\title{
Elliptic genera and characteristic q-series of superconformal field theory
}

\section{Bonora, L.}

2015-06

Bonora , L, Bytsenko , A A \& Chaichian, M 2015 , ' Elliptic genera and characteristic q-series of superconformal field theory ' , Nuclear Physics, Section B , vol. 895 , pp. 192-205 . https://doi.org/10.1016/j.nuclphysb.2015.03.030

http://hdl.handle.net/10138/203423

https://doi.org/10.1016/j.nuclphysb.2015.03.030

cc_by

publishedVersion

Downloaded from Helda, University of Helsinki institutional repository.

This is an electronic reprint of the original article.

This reprint may differ from the original in pagination and typographic detail.

Please cite the original version. 


\title{
Elliptic genera and characteristic $q$-series of superconformal field theory
}

\author{
L. Bonora ${ }^{\mathrm{a}, \mathrm{b}}$, A.A. Bytsenko ${ }^{\mathrm{c}}$, M. Chaichian ${ }^{\mathrm{d}, *}$ \\ ${ }^{a}$ International School for Advanced Studies (SISSA/ISAS), Via Bonomea 265, 34136 Trieste, Italy \\ ${ }^{\mathrm{b}}$ INFN, Sezione di Trieste, Italy \\ ${ }^{c}$ Departamento de Física, Universidade Estadual de Londrina, Caixa Postal 6001, Londrina-Paraná, Brazil \\ ${ }^{\mathrm{d}}$ Department of Physics, University of Helsinki, P.O. Box 64, FI-00014 Helsinki, Finland
}

Received 7 March 2015; accepted 31 March 2015

Available online 7 April 2015

Editor: Hubert Saleur

\begin{abstract}
We analyze the characteristic series, the $K O$ series and the series associated with the Witten genus, and their analytic forms as the $q$-analogs of classical special functions (in particular $q$-analog of the beta integral and the gamma function). $q$-Series admit an analytic interpretation in terms of the spectral Ruelle functions, and their relations with appropriate elliptic modular forms can be described. We show that there is a deep correspondence between the characteristic series of the Witten genus and $K O$ characteristic series, on one side, and the denominator identities and characters of $N=2$ superconformal algebras, and the affine Lie (super)algebras on the other. We represent the characteristic series in the form of double series using the Hecke-Rogers modular identity.

(c) 2015 The Authors. Published by Elsevier B.V. This is an open access article under the CC BY license (http://creativecommons.org/licenses/by/4.0/). Funded by SCOAP ${ }^{3}$.
\end{abstract}

\section{Introduction}

There is a deep connection between representation theory of superconformal Lie algebras, which relies on important vertex operator other. This manifests itself in the fact that $q$-series

\footnotetext{
* Corresponding author.

E-mail addresses: bonora@sissa.it (L. Bonora), aabyts@gmail.com (A.A. Bytsenko), masud.chaichian@helsinki.fi
} (M. Chaichian). 
elliptic genera can be expressed in a quite universal way in terms of $q$-analogs of the classical special functions, in particular the spectral Ruelle functions [1,2]. In this paper we would like to call the reader's attention to this connection by reviewing the mathematical aspects associated with it and certain physical applications. For the self-contentedness of the article, we shall also present a review on the subject and of the previously obtained results. Explaining the organization of our paper gives us simultaneously the opportunity to introduce its main characters.

Section 2 is devoted to collecting prototypes of elliptic genera and examples. Our aim is to show that the algebraic structure necessary to construct the elliptic genus can be expressed (in a generic way) in terms of Ruelle (Selberg-type) spectral functions of hyperbolic geometry.

In Section 3 we consider a central concept of the paper, the characteristic series of elliptic genera, a natural topological invariant which generalizes the classical genus. We will establish several results from classical analysis and among them the classical limit for characteristic $q$-series of the elliptic genera considered in the paper. We show that the characteristic series of the Witten genus and the so-called $K O$ characteristic series admit an analytic interpretation in terms of the spectral Ruelle functions. Furthermore we point out the coincidence of characteristic series as the $q$-analogs of some classical special functions, in particular $q$-analogs of the beta integral and the $q$-gamma function.

In Section 4 we concentrate on (super)denominator identities and the characters of $N=2$ superconformal algebras (Section 4.2). We represent generating functions in the form of double series using the Hecke-Rogers modular identity. The classical functional equations for the Ruelle spectral functions and the modular forms in question can be used to deduce the transformation properties of supercharacters of the $N=2$ superalgebras and denominator identities associated with the highest weight representations of affine Lie (super)algebras (see Sections 4.2 and 4.3).

Finally, in Section 5, we briefly summarize our main conclusions. The characteristic series and denominator identities can be converted into $q$-product expressions which can inherit the homology properties of appropriate superconformal Lie algebras.

To complete this introduction let us summarize our main results:

- The characteristic series ( $K O$ series and series associated with the Witten genus) have the form of $q$-analogs of classical special functions, in particular $q$-analogs of the beta integral and the gamma function. These $q$-series admit an analytic interpretation in terms of the spectral Ruelle functions and help describing their relations with appropriate elliptic modular forms.

- We show a profound correspondence between the characteristic series of the Witten genus and $K O$ characteristic series, on one side, and the denominator identities and characters of $N=2$ superconformal algebras, and the affine Lie (super)algebras $A_{1}^{(1)}$ and $\widehat{\mathfrak{g l}}(m, n)$, on the other.

- We represent the characteristic series in the form of double series using the Hecke-Rogers modular identity. The main techniques we use involve spectral functions of hyperbolic threegeometry. These machinery allows us to derive interesting new results (or rederive important old results) in superconformal field theory with its implications for three-dimensional quantum theory.

\section{Prototypes for elliptic genera}

For a holomorphic vector bundle $\mathcal{E}$ on $X$ and a formal variable $z$ we use the following definitions 


$$
\begin{array}{ll}
S_{q}(z \mathcal{E})=\bigoplus_{n \geq 0}(z q)^{n} \operatorname{Sym}^{n} \mathcal{E}, & \Lambda_{q}(z \mathcal{E})=\bigoplus_{n \geq 0}(z q)^{n} \operatorname{Alt}^{n} \mathcal{E}, \\
S_{q}(z \mathcal{E})^{\mathbb{C}}=S_{q}(z \mathcal{E}) \otimes S_{q}(\bar{z} \overline{\mathcal{E}}), & \Lambda_{q}(z \mathcal{E})^{\mathbb{C}}=\Lambda_{q}(z \mathcal{E}) \otimes \Lambda_{q}(\bar{z} \overline{\mathcal{E}}) .
\end{array}
$$

These formulas have good multiplicative properties and their elements should be understood as elements of the $K$-theory of the underlying space [3]:

$$
\begin{array}{ll}
S_{q}(\mathcal{E} \oplus \mathcal{F})=\left(S_{q} \mathcal{E}\right) \otimes\left(S_{q} \mathcal{F}\right), & S_{q}(\mathcal{E} \ominus \mathcal{F})=\left(S_{q} \mathcal{E}\right) \otimes\left(S_{q} \mathcal{F}\right)^{-1} \\
\Lambda_{q}(\mathcal{E} \oplus \mathcal{F})=\left(\Lambda_{q} \mathcal{E}\right) \otimes\left(\Lambda_{q} \mathcal{F}\right), & \Lambda_{q}(\mathcal{E} \ominus \mathcal{F})=\left(\Lambda_{q} \mathcal{E}\right) \otimes\left(\Lambda_{q} \mathcal{F}\right)^{-1} .
\end{array}
$$

In Eqs. (2.3), (2.4) we have used the facts that $\operatorname{Sym}^{n}(\mathcal{E} \oplus \mathcal{F})=\bigoplus_{j=0}^{n} \operatorname{Sym}^{j}(\mathcal{E}) \otimes \operatorname{Sym}^{n-j}(\mathcal{F})$, $\operatorname{Alt}^{n}(\mathcal{E} \oplus \mathcal{F})=\bigoplus_{j=0}^{n} \operatorname{Alt}^{j}(\mathcal{E}) \otimes \operatorname{Alt}^{n-j}(\mathcal{F})$. In the case of a line bundle $\mathcal{L}$, we have

$$
S_{q} \mathcal{L}=1 \bigoplus_{n \geq 1} q^{n} \mathcal{L}^{n}=(1 \ominus q \mathcal{L})^{-1}=\left(\Lambda_{-q} \mathcal{L}\right)^{-1},
$$

and therefore $\left(S_{q} \mathcal{E}\right)^{-1}=\Lambda_{-q} \mathcal{E}$ for any vector bundle $\mathcal{E}$, and similarly $\left(\Lambda_{q} \mathcal{E}\right)^{-1}=S_{-q} \mathcal{E}$.

Example (The Chern character). For the Chern polynomial $c(T X)=\prod_{j}\left(1+\zeta_{j}\right)$ the resulting Chern character is

$$
\begin{aligned}
\operatorname{ch}\left(\bigotimes_{n \geq 1} S_{q^{n}}\left((T X)^{\mathbb{C}}\right)\right) & =\prod_{j} \prod_{n=1}^{\infty}\left[\left(1-q^{n} e^{\zeta_{j}}\right)\left(1-q^{n} e^{-\zeta_{j}}\right)\right]^{-1} \\
& =\prod_{j}\left[\mathcal{R}\left(s=\left(1+z_{j}\right)(1-i \varrho(\tau))\right) \cdot \mathcal{R}\left(s=\left(1-z_{j}\right)(1-i \varrho(\tau))\right)\right]^{-1},
\end{aligned}
$$

where $q=\exp (2 \pi i \tau), \varrho(\tau)=\operatorname{Re} \tau / \operatorname{Im} \tau$ and $\zeta_{j}=z_{j} \log (q)$. The Ruelle zeta-functions $\mathcal{R}(s)$ is a ratio of so-called Patterson-Selberg zeta-functions (see for example [1]); it is defined for $\operatorname{Re} s \gg 1$ and can be continued to a meromorphic function on the entire complex plane $\mathbb{C}$. Its value $\mathcal{R}(0)$ computes the $L^{2}$-analytic torsion of the hyperbolic three-manifold. One has the infinite product identities $[4,1]$ :

$$
\begin{aligned}
& \prod_{n=m}^{\infty}\left(1-q^{\mu n+\varepsilon}\right)=\mathcal{R}(s=(\mu m+\varepsilon)(1-i \varrho(\tau))+1-\mu), \\
& \prod_{n=m}^{\infty}\left(1+q^{\mu n+\varepsilon}\right)=\mathcal{R}(s=(\mu m+\varepsilon)(1-i \varrho(\tau))+1-\mu+i \sigma(\tau)),
\end{aligned}
$$

where $\sigma(\tau)=(2 \operatorname{Im} \tau)^{-1}, \mu \in \mathbb{R}, m \geq 1$ and $\varepsilon, v \in \mathbb{C}$.

\section{Elliptic genera and their characteristic $q$-series}

There are various applications of modular forms in topology and physics, the elliptic genus and its generalizations being one of them. Let us recall some examples of genera:

- The Todd genus. Its characteristic series is the formal power series $z /\left(1-e^{-z}\right)=$ $\sum_{j=0}^{\infty}(-1)^{j}\left(B_{j} / j !\right) z^{j}$, where $B_{j}$ are Bernoulli numbers. The Todd genus of a stable almost complex manifold is an integer. 
- The $\widehat{A}$ genus. Its characteristic series is: $(z / 2) / \sinh (z / 2) \equiv z /\left(e^{z / 2}-e^{-z / 2}\right)$. This genus is an invariant of oriented manifolds and has the property that it assumes integral values on manifolds that admit a Spin structure.

- The Witten genus $[5,6]$ is the genus with characteristic series

$$
\begin{aligned}
& \frac{\zeta / 2}{\sinh (\zeta / 2)} \prod_{n=1}^{\infty} \frac{\left(1-q^{n}\right)^{2}}{\left(1-e^{\zeta} q^{n}\right)\left(1-e^{-\zeta} q^{n}\right)} \\
& \stackrel{\text { (by Eq. (2.7)) }}{=} \frac{\pi \tau z}{\sin (\pi \tau z)}\left[\frac{\mathcal{R}(s=1-i \varrho(\tau))}{\mathcal{R}(s=(1+z)(1-i \varrho(\tau)))}\right] \\
& \quad \times \quad\left[\frac{\mathcal{R}(s=1-i \varrho(\tau))}{(\mathcal{R}(s=(1-z)(1-i \varrho(\tau)))}\right],
\end{aligned}
$$

where $\zeta=z \log (q)$. It is an even function of the variable $z$ and defines a cobordism invariant of oriented manifolds. When applied to manifolds that admit a Spin structure it takes values in $\mathbb{Z}[[q]]$.

- In [5] a genus $\chi$ of Spin manifolds with so-called $K O$ characteristic series

$$
\sigma(L, q)=\left(L^{1 / 2}-L^{-1 / 2}\right) \prod_{n=1}^{\infty} \frac{\left(1-L q^{n}\right)\left(1-L^{-1} q^{n}\right)}{\left(1-q^{n}\right)^{2}}
$$

has been introduced. If $\tau$ is a number in the complex upper half-plane, $\varpi \in \mathbb{C}$, and $L=$ $\exp (2 \pi i \tau \varpi), \sigma(L, q)$ is a holomorphic function of $\varpi$,

$$
\begin{aligned}
\sigma(\varpi, q)= & \left(q^{\varpi / 2}-q^{-\varpi / 2}\right)\left[\frac{\mathcal{R}(s=(1+\varpi)(1-i \varrho(\tau)))}{\mathcal{R}(s=1-i \varrho(\tau))}\right] \\
& \times\left[\frac{\mathcal{R}(s=(1-\varpi)(1-i \varrho(\tau)))}{\mathcal{R}(s=1-i \varrho(\tau))}\right],
\end{aligned}
$$

which vanishes to first order of $\varpi$ at each of the points of the lattice $2 \pi i(1+\tau) \mathbb{Z}$.

Let $K[[q]]$ be a spectrum representing complex $K$-theory with coefficients extended to $\mathbb{Z}[[q]]$. Let $\Phi: X U \rightarrow K[[q]]$ be the complex orientation, where $X U\langle 2 p\rangle$ is the bordism spectrum of manifolds $X$ with complex tangent bundle and trivializations of $c_{1}, \ldots, c_{p-1}$, so $X U\langle 2\rangle=X U$ (see for detail [7]). This complex associates to a manifold $X$ of complex dimension $d$ the genus $\operatorname{Td}\left(\bigotimes_{n \geq 1} S_{q^{n}}\left((T X)-\mathbb{C}^{d}\right) \bigotimes_{n \geq 1} S_{q^{n}}\left((\overline{T X})-\mathbb{C}^{d}\right)\right)$. In addition its $K$-theory Euler class takes the form [7]

$$
\Phi(x, q)=\left(1-x^{-1}\right) \prod_{n=1}^{\infty}\left[\frac{\left(1-x q^{n}\right)\left(1-x^{-1} q^{n}\right)}{\left(1-q^{n}\right)^{2}}\right] .
$$

Eq. (3.4) (with relationship analogous to the relation between Todd genus and $\hat{A}$ genus) is a version of the Witten characteristic series. As it has been explained in [7], the orientation $(\delta \Phi)^{\sharp}$ maps a manifold $X$ of dimension $d$ to the genus

$$
\Phi\left(y^{-1}, d\right) \mathrm{Td}\left(\bigotimes_{n \geq 1} S_{q^{n}}(T X) \bigotimes_{n \geq 1} S_{q^{n}}(\overline{T X}) \bigotimes_{n \geq 1} \Lambda_{-y q^{n}}(T X) \bigotimes_{n \geq 1} \Lambda_{-y^{-1} q^{n}}(\overline{T X})\right)
$$

This is one of the standard formulas for the two-variable elliptic genus. 
Remark 3.1. We recall briefly some well known examples of vertex operator algebra bundles which have been used in the literature to study the elliptic genus and the Witten genus. If $X$ is a Riemannian manifold, then the transition functions of the complex tangent bundle $T_{\mathbb{C}} X$ lie in the special orthogonal group $S O(d)$, where $d$ is the dimension of $X$. Then $\bigotimes_{n \geq 1} S_{q^{n}}\left((T X)^{\mathbb{C}}\right)$ is a $V(1)^{S O(d)}$-bundle, where $V(1)$ is the Heisenberg vertex operator algebra of dimension $d$, with $S O(d)$ as a subgroup of $\operatorname{Aut}(V(1))$, and $V(1)^{S O(d)}$ is the set of $S O(d)$-invariants of $V(1)$, which is a vertex operator subalgebra of $V(1) . \bigotimes_{n \geq 0} \Lambda_{q^{n+1 / 2}}\left((T X)^{\mathbb{C}}\right)$ is an $L(1,0)^{S O(d)}$-bundle (we assume that $d$ is even) where $L(1,0)$ is the level one module for the affine algebra $D_{d / 2}^{(1)}$.

If $X$ is further assumed to be a spin manifold $\mathcal{S}$, then $\mathcal{S} \otimes \bigotimes_{n \geq 1} \Lambda_{q^{n}}\left((T X)^{\mathbb{C}}\right)$ is also a $L(1,0)^{S O(d)}$-bundle. If $X$ is a spin manifold and in addition the characteristic class of bundles $c_{2}(X)$ is trivial, then the Witten genus of $X$ admits a $q$-expansion as a modular form for the group $S L(2, \mathbb{Z})$. Thus, the Witten genus of $X$ can be written in terms of spectral Ruelle functions of hyperbolic geometry, as a holomorphic function on the upper half plane $\operatorname{Re} \tau>0$.

$q$-Analog of the beta integral. For further manipulations of $q$-series we need recalling some results from classical analysis and, in particular, the $q$-analog of the beta integral. The beta integral and its important generalization, the integral representation of the hypergeometric function, and their $q$-analogous are:

$$
\begin{aligned}
\int_{0}^{1} t^{p-1}(1-t)^{q-1} d t & =\frac{\Gamma(p) \Gamma(q)}{\Gamma(p+q)}(\operatorname{Re} p>0, \operatorname{Re} q>0) \\
{ }_{2} F_{1}\left[\begin{array}{c}
a, b ; z \\
c
\end{array}\right] & =\frac{\Gamma(c)}{\Gamma(b) \Gamma(c-b)} \int_{0}^{1} t^{b-1}(1-t)^{c-b-1}(1-z t)^{-a} d t \\
& =\sum_{n=0}^{\infty} \frac{(a)_{n}(b)_{n} z^{n}}{n !(c)_{n}}, \\
\int_{0}^{1} t^{\beta-1}(t q ; q)_{\alpha-1} d(q, t) & =\frac{\Gamma_{q}(\beta) \Gamma_{q}(\alpha)}{\Gamma_{q}(\alpha+\beta)}, \\
{ }_{2} \phi_{1}\left[\begin{array}{c}
q^{\alpha}, q^{\beta} ; q, z \\
q^{\gamma}
\end{array}\right] & =\frac{\Gamma_{q}(\gamma)}{\Gamma_{q}(\beta) \Gamma_{q}(\gamma-\beta)} \int_{0}^{1} \frac{t^{\beta-1}(t q ; q)_{\gamma-\beta-1}}{(x t ; q)_{\alpha}} d(q, t) \\
& =\sum_{n=0}^{\infty} \frac{(a ; q)_{n}(b ; q)_{n} z^{n}}{(q ; q)_{n}(c ; q)_{n}} .
\end{aligned}
$$

Here $(a)_{n}=a(a+1) \cdots(a+n-1),(a ; q)_{n}=\prod_{m=0}^{\infty}\left[\left(1-a q^{m}\right) /\left(1-a q^{m+n}\right)\right]=(1-a)(1-$ $a q) \cdots\left(1-a q^{n-1}\right)$, if $n$ is a nonnegative integer. The $q$-gamma function can be defined by

$$
\Gamma_{q}(z) \stackrel{\text { def }}{=} \frac{(q ; q)_{\infty}}{\left(q^{x} ; q\right)_{\infty}}(1-q)^{1-z} \equiv \prod_{n=0}^{\infty} \frac{1-q^{n+1}}{1-q^{n+z}}(1-q)^{1-z}, \quad|q|<1,
$$

and hence 


$$
\Gamma_{q}(1+z)=\prod_{n=0}^{\infty} \frac{1-q^{n+1}}{1-q^{n+z+1}}(1-q)^{-z}=\prod_{n=1}^{\infty} \frac{\left(1-q^{n}\right)\left(1-q^{n+1}\right)^{z}}{\left(1-q^{n+z}\right)\left(1-q^{n}\right)^{z}} .
$$

In [8] it has been proved that $\lim _{q \rightarrow 1^{-}} \Gamma_{q}(z)=\Gamma(z)$. Consequently $\lim _{q \rightarrow 1^{-}} \Gamma_{q}(1-z)=$ $\Gamma(1-z), \lim _{q \rightarrow 1^{-}} \Gamma_{q}(1+z)=\Gamma(1+z)$. Thus for $\operatorname{ch}\left(\bigotimes_{n \geq 1} S_{q^{n}}\left((T X)^{\mathbb{C}}\right)\right)$ in Eq. (2.6) we get

$$
\prod_{j} \prod_{n=1}^{\infty}\left(1-e^{\zeta_{j}} q^{n}\right)\left(1-e^{-\zeta_{j}} q^{n}\right)=\frac{\prod_{n=1}^{\infty}\left(1-q^{n}\right)^{2}}{\prod_{j} \Gamma_{q}\left(1+z_{j}\right) \Gamma_{q}\left(1-z_{j}\right)} .
$$

The characteristic series (3.1) (and its inverse analog (3.3)) becomes

$$
\begin{aligned}
\frac{\zeta / 2}{\sinh (\zeta / 2)} \prod_{n=1}^{\infty} \frac{\left(1-q^{n}\right)^{2}}{\left(1-e^{\zeta} q^{n}\right)\left(1-e^{-\zeta} q^{n}\right)} & =\frac{\pi \tau z}{\sin (\pi \tau z)} \prod_{n=1}^{\infty} \frac{\left(1-q^{n}\right)^{2}}{\left(1-q^{n+z}\right)\left(1-q^{n-z}\right)} \\
& =\frac{\pi \tau z}{\sin (\pi \tau z)} \Gamma_{q}(1+z) \Gamma_{q}(1-z), \\
\lim _{q \rightarrow 1^{-}} \frac{\pi \tau z}{\sin (\pi \tau z)} \Gamma_{q}(1+z) \Gamma_{q}(1-z) & =\frac{\pi \tau z}{\sin (\pi \tau z)} \Gamma(1+z) \Gamma(1-z) \\
& =\frac{\pi \tau z}{\sin (\pi \tau z)} \cdot \frac{\pi z}{\sin (\pi z)} .
\end{aligned}
$$

So far we have established several results from classical analysis and among them the classical limit for the characteristic series of the Witten genus. In particular the $q$-analog of the classical result (3.13), (3.14) is its important generalization (3.12).

Remark 3.2. Next let us consider the following integral [9]:

$$
\begin{aligned}
\mathcal{I}(a ; z) & =\int_{0}^{\infty} \frac{(1+a t)(1+a t q)\left(1+a t q^{2}\right) \cdots}{(1+t)(1+t q)\left(1+t q^{2}\right) \cdots} t^{z-1} d t \\
& =\frac{\pi}{\sin (\pi z)} \prod_{n=1}^{\infty} \frac{\left(1-q^{n-z}\right)\left(1-a q^{n-1}\right)}{\left(1-q^{n}\right)\left(1-a q^{n-z-1}\right)} .
\end{aligned}
$$

In the case $a=q^{z+1}$ we get

$$
\begin{aligned}
\mathcal{I}\left(q^{z+1} ; z\right) & =\frac{\pi}{\sin (\pi z)} \prod_{n=1}^{\infty} \frac{\left(1-q^{n+z}\right)\left(1-q^{n-z}\right)}{\left(1-q^{n}\right)^{2}} \\
& =\frac{\pi}{\sin (\pi z)}\left[\frac{\mathcal{R}(s=(1+z)(1-i \varrho(\tau)))}{\mathcal{R}(s=1-i \varrho(\tau))}\right]\left[\frac{\mathcal{R}(s=(1-z)(1-i \varrho(\tau)))}{\mathcal{R}(s=1-i \varrho(\tau))}\right] .
\end{aligned}
$$

Then the characteristic series (3.1) becomes

$$
\frac{\pi \tau z}{\sin (\pi \tau z)} \prod_{n=1}^{\infty} \frac{\left(1-q^{n}\right)^{2}}{\left(1-q^{n+z}\right)\left(1-q^{n-z}\right)}=\frac{\pi \tau z}{\sin (\pi \tau z)} \cdot \frac{\pi z}{\sin (\pi z)} \mathcal{I}\left(q^{z+1} ; z\right)^{-1} .
$$

The classical limit of (3.17) coincides with (3.14) (it is clear that $\lim _{q \rightarrow 1^{-}} \mathcal{I}\left(q^{z+1} ; z\right)^{-1}=1$ ). 


\section{Weyl character and denominator formulas}

One of the most important features of group representations is the modular invariance of their Kač-Weyl character formula, which allows us to derive many new results and to unify many important results in topology. Let us recall that the Weyl character formula in representation theory describes the characters of irreducible representations of compact Lie groups in terms of their highest weights. The precise statement is: The character of an irreducible representation $V$ of a complex semisimple Lie algebra $\mathfrak{g}$ is given by

$$
\operatorname{ch}(V)=\frac{\sum_{w \in W} \varepsilon(w) e^{w(\lambda+\rho)}}{e^{\rho} \prod_{\alpha \in \Delta_{+}}\left(1-e^{-\alpha}\right)} .
$$

Here $W$ is the Weyl group, $\Delta_{+}$is the subset of the positive roots of the root system $\Delta, \rho$ is the half sum of the positive roots, $\lambda$ is the highest weight of the irreducible representation $V$, $\varepsilon(w)$ is the determinant of the action of $w$ on the Cartan subalgebra $\mathfrak{h} \subset \mathfrak{g}$ and this is equal to $(-1)^{\ell(w)}$, where $\ell(w)$ is the length of the Weyl group element, defined to be the minimal number of reflections with respect to simple roots such that $w$ equals the product of those reflections.

In the special case of the trivial one-dimensional representation the character is 1 , so the Weyl character formula becomes the Weyl denominator formula:

$$
\sum_{w \in W} \varepsilon(w) e^{w(\rho)}=e^{\rho} \prod_{\alpha \in \Delta_{+}}\left(1-e^{-\alpha}\right) .
$$

For special unitary groups this is equivalent to the expression $\sum_{\sigma \in S_{n}} \operatorname{sgn}(\sigma) X_{1}^{\sigma(1)-1} \cdots X_{n}^{\sigma(n)-1}$ $=\prod_{1 \leq i<j \leq n}\left(X_{j}-X_{i}\right)$ i.e. the Vandermonde determinant. The Weyl character formula also holds for integrable highest weight representations of Kač-Moody algebras, in which case it is known as the Weyl-Kač character formula.

(Super)denominators. The rational exponential functions

$$
\begin{aligned}
\mathfrak{D} & =\prod_{\alpha \in \Delta_{0+}}\left(1-e^{-\alpha}\right) \prod_{\alpha \in \Delta_{1+}}\left(1+e^{-\alpha}\right)^{-1}, \\
\widehat{\mathfrak{D}} & =\prod_{\alpha \in \Delta_{0+}}\left(1-e^{-\alpha}\right) \prod_{\alpha \in \Delta_{1+}}\left(1-e^{-\alpha}\right)^{-1} \\
& =\prod_{\alpha \in \bar{\Delta}_{0+}}\left(1-e^{-\alpha}\right) \prod_{\alpha \in \Delta_{1+} \backslash \bar{\Delta}_{1+}}\left(1+e^{-\alpha}\right) \prod_{\alpha \in \bar{\Delta}_{1+}}\left(1-e^{-\alpha}\right)^{-1}
\end{aligned}
$$

are called the Weyl denominator and the Weyl superdenominator of $\mathfrak{g}$, respectively [10]. Here the set of roots $\Delta=\Delta_{0} \cup \Delta_{1}$ can be represented as a disjoint union of two subsets, called the sets of even and odd roots respectively. We introduce also the so-called affine denominator by

$$
\overline{\mathfrak{D}}=\mathfrak{D} \prod_{n \geqslant 1}\left(1-q^{n}\right)^{\ell} \prod_{\alpha \in \Delta_{0}}\left(1-e^{\alpha} q^{n}\right) \prod_{\alpha \in \Delta_{1}}\left(1+e^{\alpha} q^{n}\right)^{-1} .
$$

A denominator identity for the case of affine Lie algebras is equivalent to a Macdonald identity, while for the simplest case of the affine Lie algebra this is the Jacobi triple product identity.

Recall that in case of affine Lie algebras, $\mathfrak{g}=\mathfrak{g}(A)$, where $A$ is of finite type. The Cartan matrix of $\mathfrak{g}$ is $A=\left(a_{i j}\right)_{i, j=1, \ldots, n},\left(a_{i i}=2, a_{i j} \leq 0, a_{i j}=0\right.$ if and only if $\left.a_{j i}=0\right), a_{00}=2$ and $a_{0 i}=-2\left(\theta, \alpha_{i}\right) /\left(\alpha_{i}, \alpha_{i}\right), a_{i 0}=\left(\theta, \alpha_{i}\right) /(\theta, \theta)$, a unique maximal root $\theta \in \Delta_{+}$. The formal expression for the partition function $K$ of affine Lie algebras is [11] 


$$
\prod_{\alpha \in \triangle_{+}}\left(1-e^{-\alpha}\right)^{- \text {mult } \alpha}=\sum_{\beta \in \mathfrak{h}^{*}} K(\beta) e^{\beta},
$$

where $\mathfrak{h}$ is the Cartan subalgebra of $\mathfrak{g}, \triangle \subset \mathfrak{h}^{*}$ is the root system, $\Delta_{+}$is the set of positive roots. $K$ is defined on $\mathfrak{h}^{*}$. Since $\left(1-e^{-\alpha}\right)^{-1}=\sum_{n=0}^{\infty} e^{-n \alpha}, K(\beta)$ is the number of partitions of $\beta$ into sum of positive roots, where each root is counted with multiplicity.

\subsection{Infinite series generating functions}

The Hecke-Rogers modular form identity. It has been recognized by E. Hecke [12] and L.J. Rogers [13] that certain modular forms could be represented by combinations of the following double series:

$$
\sum_{(m, n) \in \Omega}(-1)^{\mathcal{H}(m, n)} q^{\mathcal{L}(m, n)+\mathcal{Q}(m, n)}
$$

Here $\mathcal{H}, \mathcal{L}$ are linear forms, $\mathcal{Q}$ is an indefinite quadratic form and $\Omega$ is some subset of $\mathbb{Z} \times \mathbb{Z}$. The deepest results on this topic have come from [14,11] where a number of identities in the representation theory of Kač-Moody Lie algebras have been obtained. An interesting family of modular functions satisfying Rogers-Ramanujan type identities for arbitrary affine root systems has been obtained in [15]. Extensive work in the theory of partition identities shows that basic hypergeometric series provide the generating functions for numerous families of partition identities.

Infinite double series. Let us introduce the following functions:

$$
\begin{aligned}
Q_{n, k}\left(a_{1}, a_{2}, a_{3}, a_{4}, a_{5} ; q\right) & :=(-1)^{n+k} q^{a_{1} n+a_{2} k+a_{3} n k+a_{4} n^{2}+a_{5} k^{2}}, \\
\mathcal{J}\left(a_{1}, a_{2}, a_{3}, a_{4}, a_{5} ; q\right) & :=\left(\sum_{n, k \geq 0}-\sum_{n, k<0}\right) Q_{n, k}\left(a_{1}, a_{2}, a_{3}, a_{4}, a_{5} ; q\right) .
\end{aligned}
$$

The identity

$$
\begin{aligned}
\sum_{n, k=-\infty}^{\infty} Q_{n, k \geq|2 n|}\left(\frac{1}{2}, \frac{1}{2}, 0,-\frac{3}{2}, \frac{1}{2} ; q\right) & =\sum_{n, k=-\infty ; k \geq|2 n|}^{\infty}(-1)^{n+k} q^{\left(k^{2}-3 n^{2}\right) / 2+(n+k) / 2} \\
& =\prod_{n=1}^{\infty}\left(1-q^{n}\right)^{2}
\end{aligned}
$$

was conjectured by Rogers and has been proved in $[12,11,16]$. We use the Rogers approach to present the characteristic series by combination of double series (see also [17]).

$$
\begin{aligned}
& \sum_{n, k=-\infty}^{\infty} Q_{n, k \geq|n|}\left(\frac{1}{2}, \frac{1}{2}, 0, \frac{1}{2},-\frac{3}{2} ; q\right) \stackrel{\left(1<|q|^{-1}\right)}{=} \prod_{j=1}^{\infty} \frac{\left(1-q^{j}\right)^{2}}{\left(1-q^{j-1}\right)\left(1-q^{j}\right)} \\
& \mathcal{J}\left(\frac{1}{2}, \bar{x}+\frac{1}{2}, 1, \frac{1}{2}, 0 ; q\right)=\left(\sum_{n, k \geq 0}-\sum_{n, k<0}\right) Q_{n, k}\left(\frac{1}{2}, \bar{x}+\frac{1}{2}, 1, \frac{1}{2}, 0 ; q\right) \\
&=\prod_{n=1}^{\infty} \frac{\left(1-q^{n}\right)^{2}}{\left(1+x q^{n-1 / 2}\right)\left(1+x^{-1} q^{n-1 / 2}\right)}
\end{aligned}
$$




$$
\begin{aligned}
& =\left[\frac{\mathcal{R}(s=1-i \varrho(\tau))}{\mathcal{R}(s=(\bar{x}+1 / 2)(1-i \varrho(\tau))+i \sigma(\tau)}\right] \\
& \times\left[\frac{\mathcal{R}(s=1-i \varrho(\tau))}{\mathcal{R}(s=(1-\bar{x})(1-i \varrho(\tau))+i \sigma(\tau)}\right] \text {, } \\
& \mathcal{J}\left(\frac{1}{2}, \bar{x}-\frac{1+\tau}{2 \tau}+\frac{1}{2}, 1, \frac{1}{2}, 0 ; q\right)=\prod_{n=1}^{\infty} \frac{\left(1-q^{n}\right)^{2}}{\left(1-x q^{n}\right)\left(1-x^{-1} q^{n}\right)} \\
& =\left[\frac{\mathcal{R}(s=1-i \varrho(\tau))}{\mathcal{R}(s=(1+\bar{x})(1-i \varrho(\tau)))}\right] \\
& \times\left[\frac{\mathcal{R}(s=1-i \varrho(\tau))}{\mathcal{R}(s=(1-\bar{x})(1-i \varrho(\tau)))}\right],
\end{aligned}
$$

where $\log (x)=\bar{x} \log (q)$. The right-hand side of the identity (4.13), up to the $\widehat{A}$ genus, coincides with the characteristic series (3.1).

These remarkable functions may be used for other useful identities. Indeed, formula (4.5) for the affine superdenominator $\overline{\mathfrak{D}}$ gives (see for detail Example 4.1 in [10]):

$$
\begin{aligned}
& \mathcal{J}(\bar{x}, \bar{y}, 1,0,0 ; q) \\
& =\left(\sum_{n, k \geq 0}-\sum_{n, k<0}\right)(-1)^{n+k} q^{\bar{x} n+\bar{y} k+n k} \\
& \stackrel{\left(|q|<\left|q^{\bar{x}}\right|,|y|<1\right)}{=} \prod_{j=1}^{\infty} \frac{\left(1-q^{j}\right)^{2}\left(1-x y q^{j-1}\right)\left(1-x^{-1} y^{-1} q^{j}\right)}{\left(1+x q^{j-1}\right)\left(1+x^{-1} q^{j}\right)\left(1+y q^{j-1}\right)\left(1+y^{-1} q^{j}\right)}
\end{aligned}
$$

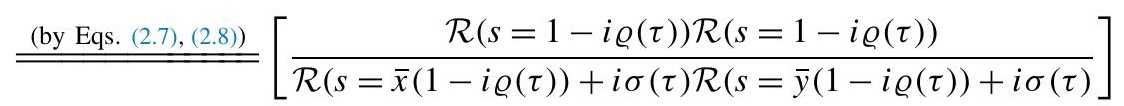

$$
\begin{aligned}
& \times \quad\left[\frac{\mathcal{R}(s=(\bar{x}+\bar{y})(1-i \varrho(\tau))+i \sigma(\tau))}{\mathcal{R}(s=(1-\bar{x})(1-i \varrho(\tau))+i \sigma(\tau))}\right] \\
& \times \quad\left[\frac{\mathcal{R}(s=(1-\bar{x}-\bar{y})(1-i \varrho(\tau))+i \sigma(\tau))}{\mathcal{R}(s=(1-\bar{y})(1-i \varrho(\tau))+i \sigma(\tau))}\right],
\end{aligned}
$$

where $\log (x)=\bar{x} \log (q), \log (y)=\bar{y} \log (q)$. This identity may be viewed as the denominator identity for the $N=4$ superconformal algebra. In the case $|q|<\left|q^{\bar{x}}\right|<1$ we also can get [10]:

$$
\mathcal{J}(\bar{x}, \bar{y}, 1,0,0 ; q)=\sum_{j \in \mathbb{Z}}(-x)^{j}\left(1+y q^{j}\right)^{-1} .
$$

Spectral functions of hyperbolic geometry and modular forms. In many problems, there are numerous instances in which we need an infinite product representation of a generating function. Let us introduce some well-known functions and their modular properties under the action of $\operatorname{SL}(2, \mathbb{Z})$.

$$
\begin{aligned}
& \varphi_{1}(q)=q^{-\frac{1}{48}} \prod_{n=1}^{\infty}\left(1-q^{n+\frac{1}{2}}\right)=q^{-\frac{1}{48}} \mathcal{R}(s=3 / 2(1-i \varrho(\tau)))=\frac{\eta_{D}\left(q^{\frac{1}{2}}\right)}{\eta_{D}(q)}, \\
& \varphi_{2}(q)=q^{-\frac{1}{48}} \prod_{n=1}^{\infty}\left(1+q^{n+\frac{1}{2}}\right)=q^{-\frac{1}{48}} \mathcal{R}(s=3 / 2(1-i \varrho(\tau))+i \sigma(\tau))
\end{aligned}
$$




$$
\begin{aligned}
& =\frac{\eta_{D}(q)^{2}}{\eta_{D}\left(q^{\frac{1}{2}}\right) \eta_{D}\left(q^{2}\right)}, \\
\varphi_{3}(q) & =q^{\frac{1}{24}} \prod_{n=1}^{\infty}\left(1+q^{n+1}\right)=q^{\frac{1}{24}} \mathcal{R}(s=2(1-i \varrho(\tau))+i \sigma(\tau))=\frac{\eta_{D}\left(q^{2}\right)}{\eta_{D}(q)},
\end{aligned}
$$

where $\eta_{D}(q) \equiv q^{1 / 24} \prod_{n \in \mathbb{Z}_{+}}\left(1-q^{n}\right)$ is the Dedekind $\eta$-function. The linear span of $\varphi_{1}(q), \varphi_{2}(q)$ and $\varphi_{3}(q)$ is $\operatorname{SL}(2, \mathbb{Z})$-invariant. Since $\varphi_{1}(q) \cdot \varphi_{2}(q) \cdot \varphi_{3}(q)=1$ we get

$$
\begin{aligned}
& \mathcal{R}(s=3 / 2(1-i \varrho(\tau)) \cdot \mathcal{R}(s=3 / 2(1-i \varrho(\tau))+i \sigma(\tau)) \\
& \quad \cdot \mathcal{R}(s=2(1-i \varrho(\tau))+i \sigma(\tau))=1 .
\end{aligned}
$$

We also have a set of useful identities:

$$
\begin{aligned}
\mathcal{R}(s=(\bar{x}+b)(1-i \varrho(\tau))+i \sigma(\tau)) \cdot \mathcal{R}(s=-(1+\bar{x}+b)(1-i \varrho(\tau))+i \sigma(\tau)) \\
=q^{-\bar{x} b-b(b+1) / 2} \mathcal{R}(s=-\bar{x}(1-i \varrho(\tau))+i \sigma(\tau)) \cdot \mathcal{R}(s=(1+\bar{x})(1-i \varrho(\tau))+i \sigma(\tau)) \\
=q^{-\bar{x}(b-1)-b(b+1) / 2} \mathcal{R}(s=(1-\bar{x})(1-i \varrho(\tau))+i \sigma(\tau)) \\
\quad \cdot \mathcal{R}(s=\bar{x}(1-i \varrho(\tau))+i \sigma(\tau)) .
\end{aligned}
$$

The classical functional equations for the Ruelle spectral functions and the modular forms in question can be used to deduce the transformation properties of the character modules of $N=2$ superalgebras.

\section{2. $N=2$ superconformal algebras}

The superconformal Lie algebras give rise to some of the most important vertex algebras. The Neveu-Schwarz (NS) algebra is the simplest among the superconformal Lie algebras. The next simplest after the NS algebra is the $N=2$ superconformal Lie algebra. The latter is a graded superalgebra spanned by a central element $\mathcal{C}$, a Virasoro formal distribution $L(z)$, an even formal distribution $J(z)$ primary with respect to $L(z)$ of conformal weight 1 , and two odd primary with respect to $L(z)$ formal distributions $G^{+}(z)$ and $G^{-}(z)$ of conformal weight $3 / 2$. The remaining operator products (OPE) can be written as follows:

$$
\begin{aligned}
& J(z) J(w) \sim \frac{\mathcal{C} / 3}{(z-w)^{2}}, \quad J(z) G^{ \pm}(w) \sim \pm \frac{G^{ \pm}(w)}{z-w}, \quad G^{ \pm}(z) G^{ \pm}(w) \sim 0, \\
& G^{+}(z) G^{-}(w) \sim \frac{\mathcal{C} / 3}{(z-w)^{3}}+\frac{J(w)}{(z-w)^{2}}+\frac{L(w)+\partial(J(w) / 2)}{z-w} .
\end{aligned}
$$

This superalgebra contains the NS subalgebra spanned by $\mathcal{C}, L(z)$ and $G(z)=G^{+}(z)+G^{-}(z)$ and the NS subalgebra spanned by $\mathcal{C}, L(z)$ and $\bar{G}(z)=i\left(G^{+}(z)-G^{-}(z)\right)[10]$.

In [18-20] the characters of the irreducible unitary representations of the $N=2$ superconformal algebras $\chi^{A, P^{ \pm}}(x, y)$ with $\mathcal{C}<1$ were computed. For the $A, P^{ \pm}$algebras they have been found to be given by

$$
\begin{gathered}
\chi_{j, k}^{(m), A}(X, Y)=X^{h_{j, k}^{A}} Y^{q_{j, k}^{A}} \Psi_{A}(Y, X) \Gamma_{j, k}^{(m)}(Y, X), \\
\chi_{j, k}^{(m), P^{ \pm}}(X, Y)=X^{h_{j, k}^{P^{ \pm}}} Y^{q_{j, k}^{P^{ \pm}}} \Psi_{P}(Y, X) \Gamma_{j, k}^{(m)}\left(Y^{ \pm 1}, X\right),
\end{gathered}
$$


where $h_{j, k}^{A}=(j k-1 / 4) / m, h_{j, k}^{P^{ \pm}}=j k / m+\mathcal{C} / 8, q_{j, k}^{A}=(j-k) / m, q_{j, k}^{P^{ \pm}}= \pm(j-k) / m, j, k \in$ $\mathbb{Z}+1 / 2, m \in \mathbb{Z}_{+}-1$ and

$$
\begin{aligned}
& \Psi_{A}(Y, X)=\prod_{n=1}^{\infty} \frac{\left(1+Y X^{n-1 / 2}\right)\left(1+Y^{-1} X^{n-1 / 2}\right)}{\left(1-X^{n}\right)^{2}} \\
& \stackrel{\text { (by Eq. (2.8)) }}{=}\left[\frac{\mathcal{R}(s=(1 / 2+\varepsilon)(1-i \varrho(\tau))+i \sigma(\tau))}{\mathcal{R}(s=1-i \varrho(\tau))}\right] \\
& \times \quad\left[\frac{\mathcal{R}(s=(1 / 2-\varepsilon)(1-i \varrho(\tau))+i \sigma(\tau))}{\mathcal{R}(s=1-i \varrho(\tau))}\right], \\
& \Psi_{P}(Y, X)=\left(Y^{1 / 2}+Y^{-1 / 2}\right) \prod_{n=1}^{\infty} \frac{\left(1+Y X^{n}\right)\left(1+Y^{-1} X^{n}\right)}{\left(1-X^{n}\right)^{2}} \\
& \stackrel{\text { (by Eq. (2.8)) }}{=} 2 \cos (\pi \tau \varepsilon)\left[\frac{\mathcal{R}(s=(1+\varepsilon)(1-i \varrho(\tau))+i \sigma(\tau))}{\mathcal{R}(s=1-i \varrho(\tau))}\right] \\
& \times \quad\left[\frac{\mathcal{R}(s=(1-\varepsilon)(1-i \varrho(\tau))+i \sigma(\tau))}{\mathcal{R}(s=1-i \varrho(\tau))}\right], \\
& \Gamma_{j, k}^{(m)}(Y, X)=Y \sum_{n=-\infty}^{\infty} X^{m n^{2}+(j+k) n}\left(\frac{1}{Y+X^{m n+j}}+\frac{1}{Y+X^{-m n-k}}\right) .
\end{aligned}
$$

In Eqs. (4.24) and (4.25) $X=\exp (2 \pi i \tau) \equiv q, \log (Y)=\varepsilon \log (q)$. There is a profound correspondence between the characteristic series for the elliptic genus and the characters (and denominator identities) for $N=2$ superconformal algebras $A, P^{ \pm}$:

$$
\begin{gathered}
\mathcal{J}\left(\frac{1}{2}, \bar{x}+\frac{1}{2}, \frac{1}{2}, 1,0 ; q\right)=\left(\sum_{n, k \geq 0}-\sum_{n, k<0}\right) q^{\frac{1}{2} n(n+1)+\left(n+\frac{1}{2}\right) k+\bar{x} k} \\
=\prod_{n=1}^{\infty} \frac{\left(1-q^{n}\right)^{2}}{\left(1+x q^{n-1 / 2}\right)\left(1+x^{-1} q^{n-1 / 2}\right)} \\
\frac{\text { (by Eq. (4.24)) }}{=} \Psi_{A}^{-1}(Y=x, X=q), \\
\Psi_{P}(Y=-x, X=q) \frac{\text { (by Eq. (3.4)) }}{=} i(x+1) \Phi(x, q), \\
\Psi_{P}(Y=-L, X=q) \frac{\text { (by Eq. (3.2)) }}{=} i \sigma(L, q) .
\end{gathered}
$$

\subsection{Affine (super)algebras, denominator identities}

For the affine Lie algebra $A_{1}^{(1)}$ computing the partition function $K(\beta)$ (see Section 4, Eq. (4.6)) in low rank is equivalent to the following identity [11]:

$$
\prod_{n=0}^{\infty}\left(1-x q^{n}\right)^{-1}\left(1-x^{-1} q^{n+1}\right)^{-1}=\frac{\sum_{n \in \mathbb{Z}}(-1)^{n}\left(1-x q^{n}\right)^{-1} q^{(1 / 2) n(n+1)}}{\prod_{n=1}^{\infty}\left(1-q^{n}\right)^{2}} .
$$


Eq. (4.30) then gives the denominator identity for the affine Lie algebra $A_{1}^{(1)}$ and also for the affine superalgebra $\widehat{\mathfrak{g l}}(1,1)^{1}$

$$
\begin{aligned}
& \prod_{k=1}^{\infty} \frac{\left(1-q^{k}\right)^{2}}{\left(1-x q^{k}\right)\left(1-x^{-1} q^{k+1}\right)} \\
& \quad=\frac{\mathcal{R}^{2}(s=1-i \varrho(\tau))}{\mathcal{R}(s=(1-\bar{x})(1-i \varrho(\tau))) \cdot \mathcal{R}(s=(2+\bar{x})(1-i \varrho(\tau)))} .
\end{aligned}
$$

Such identity can be obtained as a specialization of Ramanujan's summation formula for the bilateral hypergeometric function ${ }_{1} \psi_{1}$. Recall that the general bilateral hypergeometric function $r \psi_{s}(z)$ has the form [22]

$$
r \psi_{s}(z)=\left[\begin{array}{l}
a_{1}, a_{2}, \ldots, a_{r} \\
b_{1}, b_{2}, \ldots, b_{s}
\end{array} ; q, z\right]=\sum_{n=-\infty}^{\infty} \frac{\left(a_{1}, a_{2}, \ldots, a_{r} ; q\right)_{n}}{\left(b_{1}, b_{2}, \ldots ; q\right)_{n}}(-1)^{(s-r) n} q^{(s-r) n(n-1) / 2} z^{n}
$$

it assumes that each term of this series is correctly defined. The ${ }_{1} \psi_{1}(a, b ; q, z q)$-summation formula, which is due to Ramanujan, may be written as

$$
{ }_{1} \psi_{1}(a, b ; q, z q)=\frac{(q, b / a, a z, q / a z ; q)_{\infty}}{(b, q / a, z, b / a z ; q)_{\infty}}, \quad|b / a|<|z|<1 .
$$

Making use replacing $x \mapsto t q^{1 / 2}, \log x=\bar{x} \log q \mapsto \log t=(\bar{t}-1 / 2) \log q$ in Eq. (4.31) we obtain the denominator identity for the $N=2$ superconformal algebra (cf. Eqs. (4.12) and (4.13))

$$
\begin{aligned}
\mathcal{J}\left(\frac{1}{2}, \bar{t}-\frac{1+\tau}{2 \tau}+\frac{1}{2}, 1, \frac{1}{2}, 0 ; q\right) & =\prod_{n=1}^{\infty} \frac{\left(1-q^{n}\right)^{2}}{\left(1-t q^{n}\right)\left(1-t^{-1} q^{n}\right)} \\
& =(1-t)\left(1-t^{-1} q\right) \sum_{n \in \mathbb{Z}}(-1)^{n} \frac{q^{(1 / 2) n(n+1)}}{1-t q^{n}}
\end{aligned}
$$

\section{Concluding remarks}

In recent years $q$-series have attracted new interest in research areas such as topological field theory and Lie algebras, and have stimulated new developments in mathematical areas where $q$-series are familiar objects. The relationship between Lie algebras and combinatorial identities (the Euler identity, as one of its famous examples) was first discovered by Macdonald. A general procedure for proving combinatorial identities is based on the Euler-Poincaré formula. Usually the Euler-Poincaré formula applies to chain complexes of finite dimensional Lie algebras. In the infinite dimensional case matters can be fixed up by considering polygraded Lie algebras.

The partition functions can indeed be converted into product expressions; certain formulas for the partition functions (and Poincaré polynomials) are associated with dimensions of homologies of appropriate topological spaces and linked to generating functions and elliptic genera. Note that

\footnotetext{
1 The boson-fermion correspondence based on the Lie algebra $\widehat{\mathfrak{g l}}(1)$ leads to the denominator identity for $\widehat{\mathfrak{g l}}(2)$, whereas the super boson-fermion correspondence based on the Lie superalgebra $\widehat{\mathfrak{g l}}(1,1)$ produces the denominator identity for $\widehat{\mathfrak{g l}}(2,1)$. This parallel has been generalized for pairs of affine superalgebras $\widehat{\mathfrak{g l}}(m, n)$ and $\widehat{\mathfrak{g l}}(m+1, n)$ in $[21]$.
} 
this conclusively explains the sequence of dimensions (distinguished powers) of the simple Lie algebras (see Section 4, Eq. (4.6)).

A set of combinatorial identities can be obtained by applying to subalgebras of Kač-Moody algebras. Recall the construction of these subalgebras based on the Cartan matrix (see Section 4). From the point of view of the applications, homologies associated with these subalgebras constitute the technical basis of the proof of the combinatorial identities of Euler-Gauss-JacobiMacDonald.

In various applications of conformal field theory, such as the Witten genus and its characteristic series, $K O$ characteristic series, we have shown that $q$-series can be expressed in a quite universal way in terms of classical special functions and Ruelle spectral functions related to the congruence subgroup $S L(2 ; \mathbb{Z})$. These mathematical tools allow us to derive interesting new results (and important old results) in particular in superconformal field theory.

Given the significance of the Ruelle spectral function for the three dimensional hyperbolic geometry, this is a clear manifestation of the remarkable link that exists between all the above and hyperbolic three-geometry. More to the point, the link between two- and three-dimensional quantum models manifests itself in the fact that appropriate $q$-series (such as the characteristic series, the denominator identities, the characters of superconformal and affine Lie algebras) can be expressed in a quite universal way in terms of spectral functions of hyperbolic geometry. Clarifying this connection is an intriguing challenge for the future.

\section{Acknowledgements}

A.A.B. would like to acknowledge the Conselho Nacional de Desenvolvimento Científico e Tecnológico (CNPq, Brazil) and Coordenação de Aperfeiçoamento de Pessoal de Nível Superior (CAPES, Brazil) for financial support. The support of the Academy of Finland under the Project No. 272919 is gratefully acknowledged.

\section{References}

[1] A.A. Bytsenko, M. Chaichian, R.J. Szabo, A. Tureanu, Quantum black holes, elliptic genera and spectral partition functions, Int. J. Geom. Methods Mod. Phys. 11 (2014) 1450048, 42 pp. arXiv:1308.2177 [hep-th].

[2] L. Bonora, A.A. Bytsenko, M.E.X. Guimarães, Generalized $q$-deformed correlation functions as spectral functions of hyperbolic geometry, Eur. Phys. J. C 74 (2014) 2976, 8 pp. arXiv:1405.4717 [hep-th].

[3] M. Ando, E. Sharpe, Elliptic genera of Landau-Ginzburg models over nontrivial spaces, Nucl. Phys. B 414 (1994) 191-212, arXiv:hep-th/9306096v2.

[4] L. Bonora, A.A. Bytsenko, Partition functions for quantum gravity, black holes, elliptic genera and Lie algebra homologies, Nucl. Phys. B 852 (2011) 508-537, arXiv:1105.4571 [hep-th].

[5] E. Witten, Elliptic genera and quantum field theory, Commun. Math. Phys. 109 (1987) 525-536.

[6] E. Witten, The index of the Dirac operator in loop space, in: P.S. Landweber (Ed.), Elliptic Curves and Modular Forms in Algebraic Topology, in: Lect. Notes Math., vol. 1326, Springer-Verlag, New York, 1988, pp. 161-181.

[7] M. Ando, C.P. French, N. Ganter, The Jacobi orientation and the two-variable elliptic genus, Algebr. Geom. Topol. 8 (2008) 493-539, arXiv:math/0605554.

[8] W. Gosper, A calculus of series rearrangements, in: J.F. Traub (Ed.), Algorithms and Complexity, Academic Press, 1976, pp. 121-151.

[9] R. Askey, Ramanujan's extensions of the gamma and beta functions, Am. Math. Mon. 87 (1980) 346-359.

[10] V.G. Kač, M. Wakimoto, Integrable highest weight modules over affine superalgebras and number theory, Prog. Math. 123 (1994) 415-456, arXiv:hep-th/9407057.

[11] V.G. Kač, D.H. Peterson, Infinite-dimensional Lie algebras, theta functions and modular forms, Adv. Math. 53 (1984) 125-264.

[12] E. Hecke, Über den Zusammenhang zwischen elliptischen Modulfunktionen und indefiniten quadratischen Formen, Math. Werke, Vandenhoeck and Ruprecht, Göttingen, 1959, pp. 418-427. 
[13] L.J. Rogers, On the expansion of some infinite products, Proc. Lond. Math. Soc. 24 (1893) 337-352.

[14] V.G. Kač, D.H. Peterson, Affine Lie algebras and Hecke modular forms, Bull. Am. Math. Soc. 3 (1980) 1057-1061.

[15] I. Cherednik, B. Feigin, Rogers-Ramanujan type identities and Nil-DAHA, Adv. Math. 248 (2013) 1059-1088, arXiv:1209.1978v4 [math.QA].

[16] D.M. Bressound, Hecke modular forms and q-Hermite polynomials, Ill. J. Math. 30 (1986) 185-196.

[17] G.E. Andrews, $q$-Series: Their Development and Application in Analysis, Number Theory, Combinatorics, Physics, and Computer Algebra, Expository Lectures from the CBMS Regional Conference, vol. 66, AMS, Providence, Rh. I., 1986.

[18] Y. Matsuo, Character formula of $c<1$ unitary representation of $N=2$ superconformal algebra, Prog. Theor. Phys. 77 (1987) 793-797.

[19] V.K. Dobrev, Characters of the unitarizable highest weight modules over the $N=2$ superconformal algebras, Phys. Lett. B 186 (1987) 43-51, arXiv:0708.1719 [hep-th].

[20] E.B. Kiritsis, Character formulae and the structure of the representations of the $N=1, N=2$ superconformal algebras, Int. J. Mod. Phys. A 3 (1988) 1871, 38 pp.

[21] V.G. Kač, M. Wakimoto, Integrable highest weight modules over affine superalgebras and Appell's function, Commun. Math. Phys. 215 (2001) 631-682, arXiv:math-ph/0006007v1.

[22] G. Gasper, M. Rahman, Basic hypergeometric series, in: G.-C. Rota (Ed.), Encyclopedia of Mathematics and Its Applications, vol. 35, Cambridge University Press, 1990. 